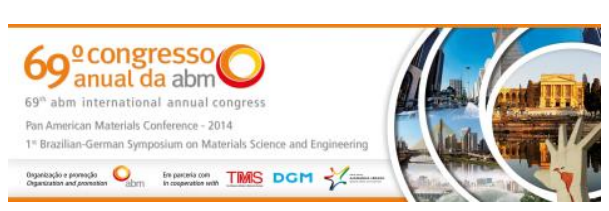

Tema: Engenharia de superfície

\title{
AVALIAÇÃO TRIBOLÓGICA DO AÇO AISI 1045 SUBMETIDO A TRATAMENTO TERMOQUÍMICO DE BORETAÇÃO MULTICOMPONENTE*
}

\author{
Claudinei Joaquim Franco ${ }^{1}$ \\ Anael Preman Krelling ${ }^{2}$ \\ Julio Cesar Giubilei Milan ${ }^{3}$ \\ César Edil da Costa ${ }^{3}$
}

\section{Resumo}

O uso de tratamentos de superfície proporciona melhoria das propriedades tribológicas dos materiais, possibilitando a utilização de materiais comuns, de menor custo e com alta tenacidade, porem menos duros, em condições que exigem alto desempenho da região de superfície. Os tratamentos termoquímicos podem aumentar o desempenho da superfície de forma mais simples e com menor custo quando comparado a processos de deposição de filmes finos. Neste trabalho foram realizados tratamentos termoquímicos de boretação, nitretação sólida e boretação multicomponente em aço AISI 1045 com o objetivo de melhorar o comportamento tribológico. O tratamento de boretação multicomponente consistiu de boretação seguido de nitretação, ambos realizados por via sólida. Foram realizados ensaios de desgaste por deslizamento do tipo pino-sobre-disco com contra corpo de WCCo. Os resultados mostraram o melhor desempenho das amostras apenas boretadas.

Palavras-chave: Boretação multicomponente; AISI 1045; Comportamento tribológico; Desgaste por deslizamento.

\section{TRIBOLOGICAL EVALUATION OF AISI 1045 STEEL SUBMITTED TO MULTICOMPONENT BORIDING THERMOCHEMICAL TREATMENT}

\begin{abstract}
The use of surface treatments provides improved tribological properties of materials, enabling the use of standard materials, with lower cost and higher toughness, but less hard, in conditions that require high-performance of surface. Compared to surface deposition of thin films, thermochemical processes can improve the surface properties in a simpler and cheaper manner. In this work, solid boriding, nitriding and multicomponent boriding thermochemical treatments were performed on AISI 1045 with the aim of improve the tribological behavior. Multicomponent boriding thermochemical treatment consisted of boriding followed by nitriding, both made in solid mediums. Pin-on-disc wear test were carried out with WCCo counterbody. The results showed a better performance of borided samples.
\end{abstract}

Keywords: Multicomponent boriding; AISI 1045; Tribological behavior; Sliding wear.

1 Eng. Mecânico, Mestrando, Departamento de Engenharia Mecânica, Universidade do Estado de Santa Catarina (UDESC), Joinville, SC, Brasil.

2 Eng. Mecânico, Doutorando, Prof., Instituto Federal de Santa Catarina (IFSC), Campus Joinville, SC, Brasil.

3 Eng. Mecânico, Dr., Professor associado, Departamento de Engenharia Mecânica, Universidade do Estado de Santa Catarina, Joinville, SC, Brasil.

\footnotetext{
* Contribuição técnica ao 69ำ Congresso Anual da ABM - Internacional e ao 14ํㅡㄹ ENEMET - Encontro Nacional de Estudantes de Engenharia Metalúrgica, de Materiais e de Minas, 21 a 25 de julho de 2014, São Paulo, SP, Brasil.
} 


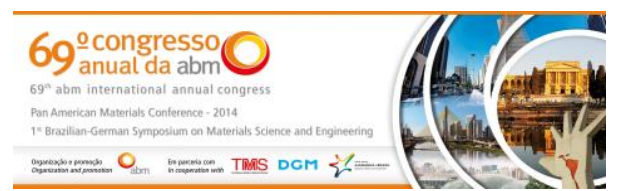

\section{INTRODUÇÃO}

Com o desenvolvimento industrial houve a consequente busca por materiais mais sofisticados e resistentes. Diante disso, tem-se tentado melhorar as características superficiais e como obter as melhores propriedades para essas superfícies, reduzindo os custos para sua obtenção. A área de engenharia de superfície abrange uma vasta gama de processos como: tratamentos mecânicos, químicos, térmicos, por bombardeamento de íons, por difusão, entre outros [1]. Nesse sentido, os tratamentos termoquímicos de boretação e nitretação são boas alternativas para a melhoria das propriedades superficiais de materiais metálicos.

Boretação é um tratamento termoquímico no qual átomos de boro se difundem para o interior da matriz metálica. Por ser de pequeno tamanho, os átomos de boro difundem-se em uma variedade de materiais como metais ferrosos, ligas de níquel e cobalto, ligas refratárias, ligas de titânio e materiais sinterizados, etc. [2-4].

O tratamento termoquímico de boretação é geralmente aplicado na indústria em ligas ferrosas para melhorar sua dureza superficial e resistência ao desgaste [5-8]. A boretação em aços, tipicamente, ocorre na faixa de temperaturas entre 840 e $1050^{\circ} \mathrm{C}[9,10]$ e pode ser realizada em uma variedade de meios como: pós, sais, óxidos fundidos, gases e pastas [11].

A difusão de átomos de boro no interior da matriz metálica leva à formação de boretos de ferro FeB e Fe2B, além de outros boretos de elementos de liga do substrato quando a boretação é feita, por exemplo, em aços ferramenta. Apesar de ser aplicada para melhoria das propriedades superficiais de peças metálicas, a boretação leva à fragilização da superfície devido ao extremo aumento de dureza. Assim, é necessário que o tratamento de boretação seja modificado [12]. Como linha futura para o desenvolvimento da técnica de boretação está a formação de camadas de boretos de múltiplos elementos ou camadas compósitas [13].

A nitretação, por sua vez, é um tratamento termoquímico, no qual o nitrogênio atômico é introduzido na superfície do aço. Algumas técnicas de nitretação são largamente utilizadas industrialmente para aumentar a dureza, resistência ao desgaste e corrosão e limite de fadiga [14].

Os precipitados de nitretos formados na superfície metálica consistem de diferentes fases como $\mathrm{Fe}_{4} \mathrm{~N}-\mathrm{y}^{\prime}$ e $\mathrm{Fe}_{2-3} \mathrm{~N}-\varepsilon$ e outros precipitados na forma $\mathrm{M}_{\mathrm{x}} \mathrm{N}_{\mathrm{y}}$. A mistura de nitretos do tipo $\mathrm{Fe}_{4} \mathrm{~N}-\gamma^{\prime}$ e $\mathrm{Fe}_{2-3} \mathrm{~N}-\varepsilon$ é chamada de camada branca, enquanto a camada de difusão é uma zona de solução sólida rica em nitrogênio, com alguns precipitados [14,15].

O tratamento termoquímico de nitretação pode ser realizado em uma grande variedade de meios, quais sejam: líquida, gasosa [16], plasma [15] e sólida [17]. Dentre esses processos, a nitretação por via sólida é um processo simples que utiliza um granulado sólido rico em nitrogênio. Quando aquecido este granulado libera nitrogênio, que se difunde a partir da superfície metálica, formando nitretos. Não há, nesse processo, a necessidade de controle atmosférico e o tratamento pode ser realizado em fornos tipo mufla [18].

A junção das duas técnicas expostas, boretação e nitretação, leva ao desenvolvimento de outro processo de tratamento superficial chamada boronitretação. Neste tipo de tratamento, o material é submetido a tratamento termoquímico de boretação sendo posteriormente nitretado. Como consequência destes processos camadas de nitretos de boro ou camadas de boretos-nitretos podem ser formadas [19].

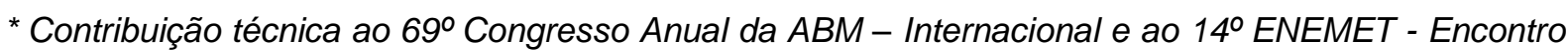
Nacional de Estudantes de Engenharia Metalúrgica, de Materiais e de Minas, 21 a 25 de julho de 2014, São Paulo, SP, Brasil.
} 


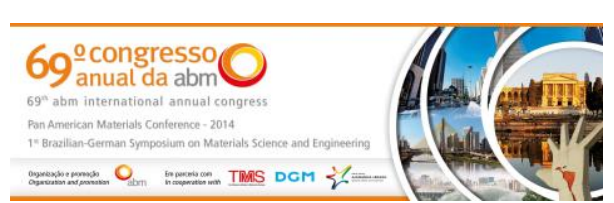

O objetivo deste trabalho, diante do exposto, é o de avaliar o comportamento tribológico de um aço comum ao carbono submetido a tratamento termoquímico de boro-nitretação. Os resultados tribológicos são comparados com amostras apenas boretadas ou nitretadas. Além disso, a composição e morfologia das camadas obtidas também serão discutidas.

\section{MATERIAIS E MÉTODOS}

Para a realização deste trabalho foi utilizado o aço comum ao carbono AISI 1045. A Tabela 1 apresenta química deste material.

Amostras foram cortadas com $6 \mathrm{~mm}$ de espessura, a partir de barras com $25 \mathrm{~mm}$ de diâmetro. Elas foram lixadas e polidas até $1 \mu \mathrm{m}$ e submetidas a tratamentos termoquímicos de boretação sólida, nitretação sólida e um tratamento de boretação seguido de nitretação, denominado boretação multicomponente.

Tabela 1. Composição química das amostras

\begin{tabular}{ccccc}
\hline & $\%$ C & $\%$ Mn & $\% \mathrm{P}$ & $\%$ S \\
\hline AISI 1045 & 0,47 & 0,80 & 0,036 & 0,02 \\
\hline Fonte: Espectroscopia de emissão óptica.
\end{tabular}

A boretação sólida foi realizada de acordo com dados e procedimento da literatura [7] com pó comercial da marca Ekabor 1-V2®. Foi utilizada uma temperatura de $1000^{\circ} \mathrm{C}$ por 2 horas. Os tratamentos de nitretação sólida foram realizados com um granulado comercial Turbonit k- $20 \AA$ com condições descritas na literatura [18], temperatura de $560^{\circ} \mathrm{C}$ por 10 horas.

Todos os tratamentos termoquímicos foram realizados em um forno tipo mufla, no Laboratório de Tratamentos Térmicos do Departamento de Engenharia Mecânica da Universidade do Estado de Santa Catarina, UDESC.

O tratamento termoquímico denominado de boretação multicomponente consistiu em submeter as amostras ao processo de boretação e, em seguida, à nitretação sólida, nas mesmas condições descritas. Previamente a realização da nitretação as amostras foram resfriadas ao ar.

Para avaliação das propriedades tribológicas, as amostras foram submetidas ao ensaio de desgaste por deslizamento do tipo pino-sobre-disco, a seco, de acordo com a norma ASTM G99/2010 [20]. O contra-corpo utilizado foi uma esfera de WCCo (metal duro) com 6,0 mm de diâmetro. A carga aplicada foi de $10 \mathrm{~N}$, a velocidade de deslizamento de $0,2 \mathrm{~m} / \mathrm{s}$ e a distância de deslizamento de $500 \mathrm{~m}$.

O desgaste foi avaliado através do volume de material removido que foi obtido com o auxílio de um Perfilômetro CV- 2000 Contrace Mitutoyo e uma rotina computacional. O coeficiente de atrito foi monitorado pelo tribômetro através da medição da força de atrito.

Para caracterização da camada boretada, as amostras foram submetidas à microscopia ótica, a microscopia eletrônica de varredura, MEV, difração de raios $x$ e ensaio de microdureza Vickers. A observação das trilhas de desgaste foi feita por MEV.

\section{RESULTADOS E DISCUSSÃO}

A Figura 1 mostra o difratograma de raios-X da amostra na qual foi realizado o tratamento termoquímico denominado boretação multicomponente. Neste

\footnotetext{
* Contribuição técnica ao 69ำ Congresso Anual da ABM - Internacional e ao 14ํㅡㄹ ENEMET - Encontro Nacional de Estudantes de Engenharia Metalúrgica, de Materiais e de Minas, 21 a 25 de julho de 2014, São Paulo, SP, Brasil.
} 
difratograma pode se observar a formação de boretos, $\mathrm{FeB}$ e $\mathrm{Fe}_{2} \mathrm{~B}$, e de nitretos $\mathrm{Fe}_{2} \mathrm{~N}$ e BN, comprovando a ação tanto da boretação como da nitretação.

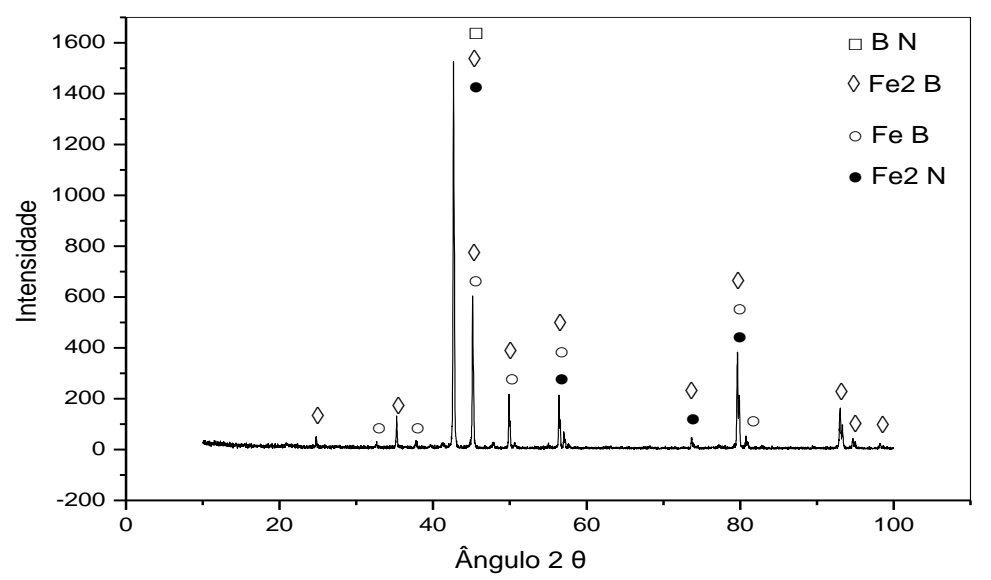

Figura 1. Difratograma de raios-X da amostra submetida à boretação multicomponente.

A Figura 2 mostra as micrografias do aço AISI 1045, obtidas por microscopia eletrônica de varredura, das amostras submetidas aos tratamentos de (a) boretação, (b) nitretação, (c) detalhe da camada branca e (d) boretação multicomponentes.

(a)

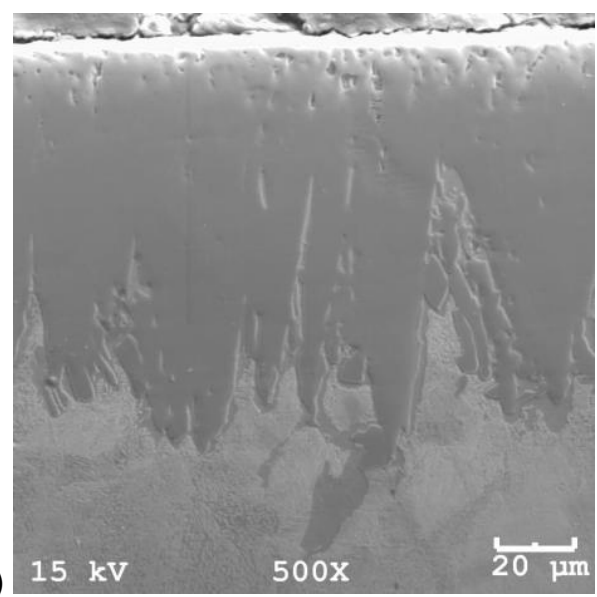

(c)

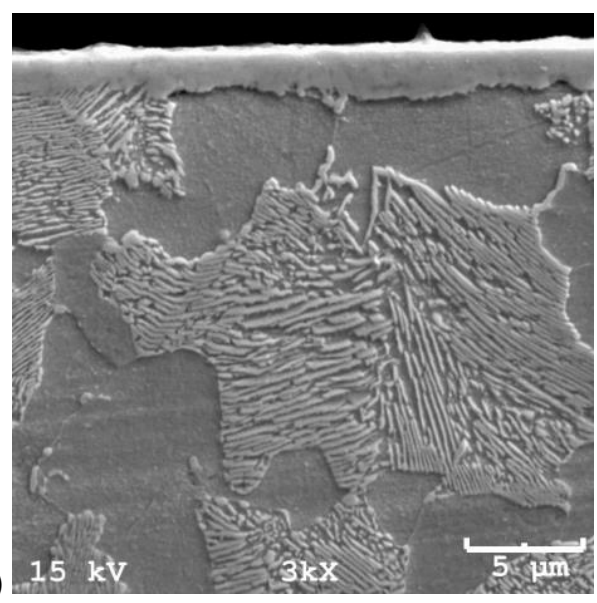

(b)

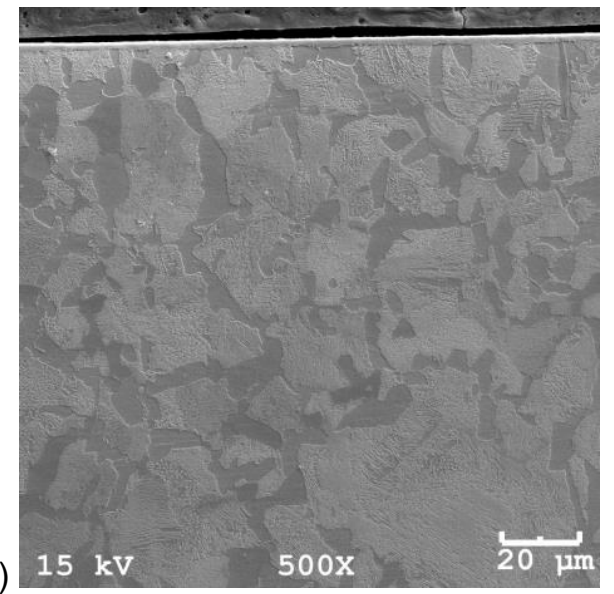

(d)

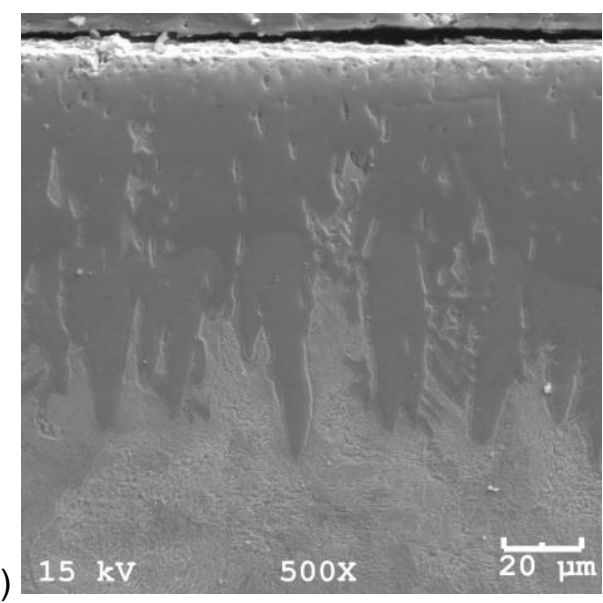

Figura 2. Micrografias, obtidas por MEV, da seção transversal das amostras submetidas a tratamentos de (a), boretação, (b) nitretação, (c) detalhe da camada de compostos da amostra nitretada e (d) boretação multicomponente.

* Contribuição técnica ao 69 Congresso Anual da ABM - Internacional e ao 14ํㅡㄹ ENEMET - Encontro Nacional de Estudantes de Engenharia Metalúrgica, de Materiais e de Minas, 21 a 25 de julho de 2014, São Paulo, SP, Brasil. 


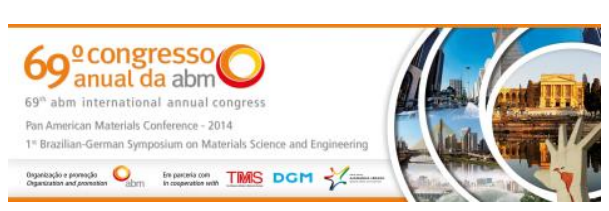

Observa-se, nas micrografias das amostras submetidas a boretação e a boretação multicomponente, a morfologia dente de serra, característica da boretação. A espessura da camada foi semelhante nos dois tratamentos, atingindo aproximadamente $100 \mu \mathrm{m}$. Na superfície da amostra submetida a boretação multicomponente nota-se um aspecto distinto daquele da amostra boretada, podendo ser uma camada de nitretos com espessura irregular, o que torna difícil a sua determinação.

Já na amostra nitretada, Figuras 2 (b) e 2 (c), nota-se nitidamente a camada de compostos bem homogênea sobre a superfície com espessura de aproximadamente $3 \mu \mathrm{m}$. Porém, não é possível identificar nem quantificar a espessura da zona de difusão.

O perfil de microdureza da seção transversal das amostras, Figura 3, comprova a espessura das camadas. Nota-se, principalmente na boretação multicomponente, uma elevação da microdureza a partir da superfície, com posterior queda e, então, valores semelhantes aos da amostra apenas boretada. Na Figura 4 observa-se ainda que a boretação multicomponente proporcionou uma dureza um pouco inferior na superfície das amostras, em comparação com a boretação. É possível comprovar a existência de uma camada de difusão através do perfil de microdureza da amostra nitretada. A microdureza sofreu uma redução a partir da superfície e atingiu valores semelhantes ao material sem tratamento a partir de $50 \mu \mathrm{m}$.

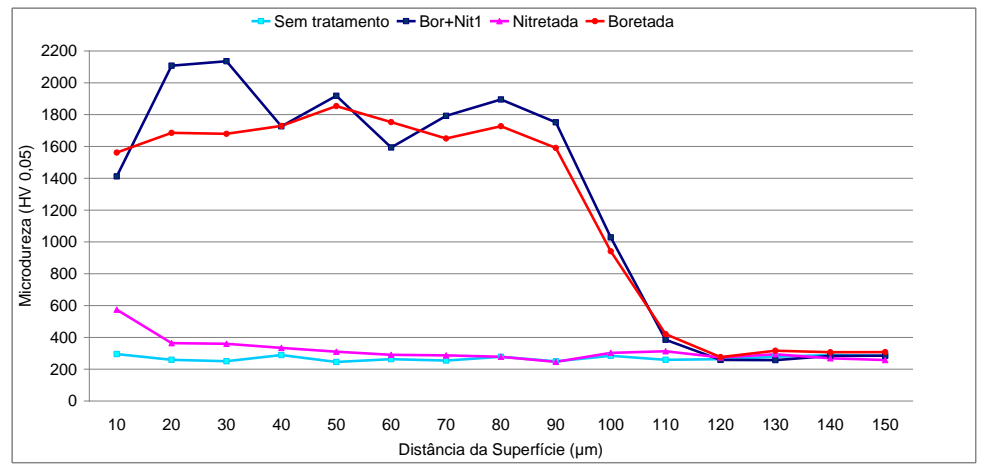

Figura 3. Perfil de microdureza da seção transversal, da seção transversal das amostras submetidas a tratamentos de boretação, nitretação e boretação multicomponente.

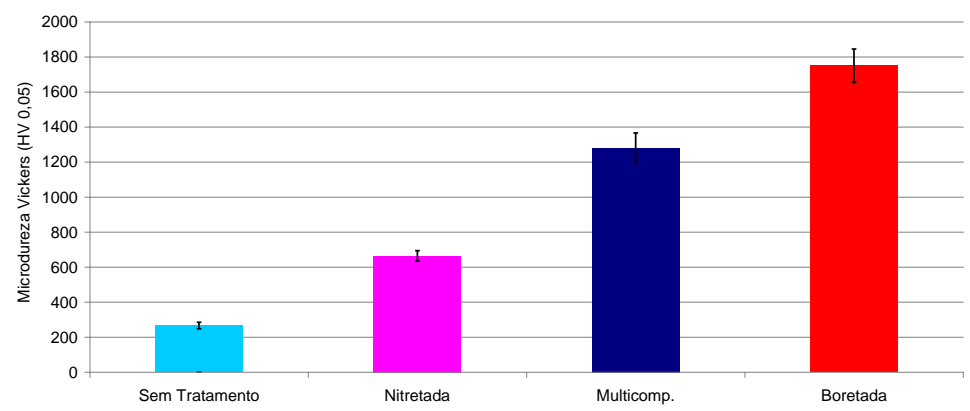

Figura 4. Valores médios de microdureza Vickers $(H V 0,05)$ da superfície das amostras após os tratamentos termoquímicos.

O valor do coeficiente de atrito monitorado durante o ensaio de desgaste é apresentado na Figura 5 para a condição boretada multicomponente. $O$ comportamento em todas as condições foi semelhante, porém, as condições nitretada e multicomponente apresentaram um valor médio de coeficiente de atrito

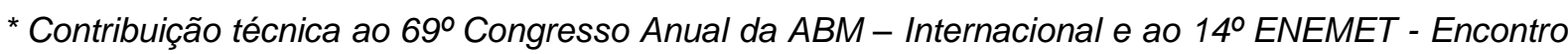
Nacional de Estudantes de Engenharia Metalúrgica, de Materiais e de Minas, 21 a 25 de julho de 2014, São Paulo, SP, Brasil.
} 


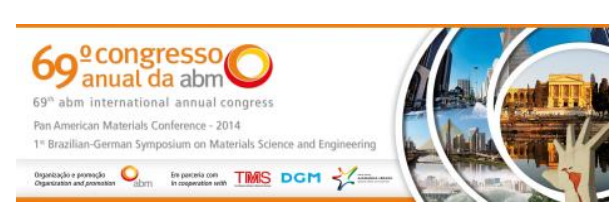

maior desgaste das três condições. Foi o tratamento que produziu a camada com menor espessura e menor dureza. A amostra tratada apenas com a boretação apresentou o melhor desempenho, apresentando volume de material removido quase 3 vezes menor que a condição boretada multicomponente, apesar da maior dureza obtida neste último tratamento. A camada da amostra submetida a boretação multicomponente apresentou um maior trincamento durante os ensaios, Figura 7, o que pode ter contribuído para o maior desgaste em relação a amostra apenas boretada.
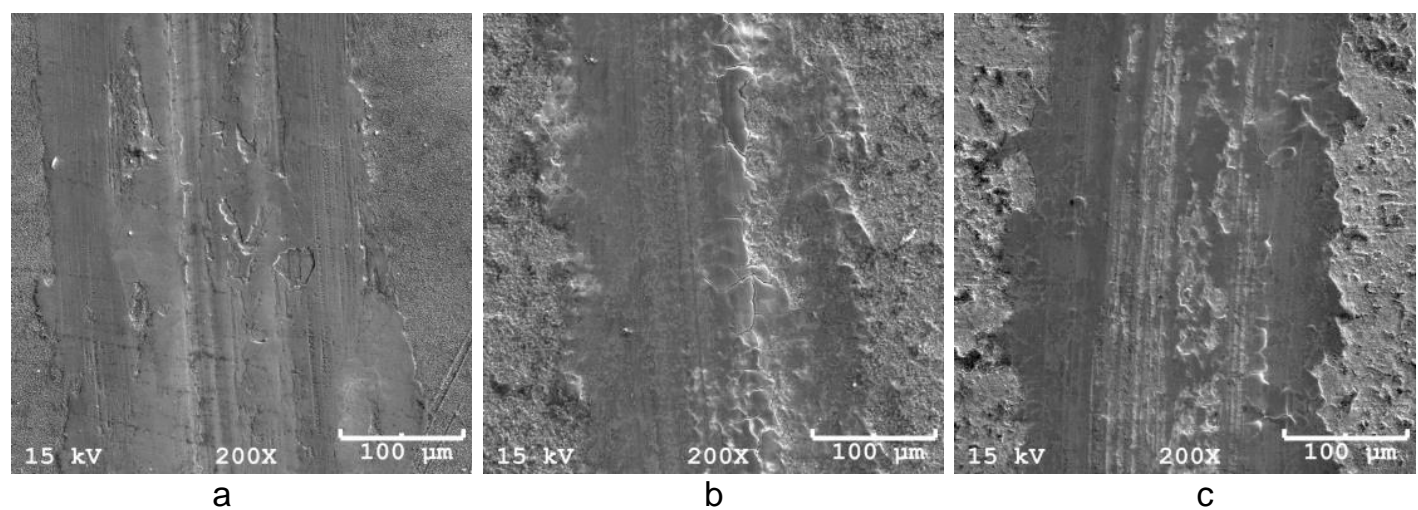

Figura 7. Aspecto das trilhas de desgaste após os ensaios de desgaste por deslizamento do tipo pino sobre disco, nas amostras submetidas à: (a) boretação, (b) boretação multicomponente e (c) nitretação sólida.

\section{CONCLUSÃO}

Com base nos resultados obtidos pode se concluir que:

- $\quad$ Os três tratamentos termoquímicos possibilitaram a formação de camadas superficiais com maiores durezas que os substratos.

- $\quad$ A boretação multicomponente produziu uma camada composta de boretos e nitretos e proporcionou a camada com maior dureza das três condições.

- $\quad$ O coeficiente de atrito atingiu valores menores nas amostras submetidas a nitretação e a boretação multicomponente.

- A condição só boretada apresentou os menores volumes de material removido, quase 3 vezes menor que a condição boretada multicomponente e pouco mais de 6 vezes a condição nitretada.

- A camada da amostra boretada multicomponente apresentou maior trincamento nos ensaios de desgaste por deslizamento.

\section{Agradecimentos}

Os autores agradecem à Capes pela bolsa de mestrado concedida.

\section{REFERÊNCIAS}

1 Almeida E. Surface treatments and coatings for metals. A general overview. 1. Surface treatments, surface preparation, and the nature of coatings. Industrial and Engineering Chemistry Research, 2001; 40(1): 3-14.

2 Atik $\mathrm{E}$, Yunker $\mathrm{U}$, Meriç $\mathrm{C}$. The effects of conventional heat treatment and boronizing on abrasive wear and corrosion of SAE 1010 , SAE 1040 , D2 and 304 steels. Tribology International, 2003; 36: 155-161.

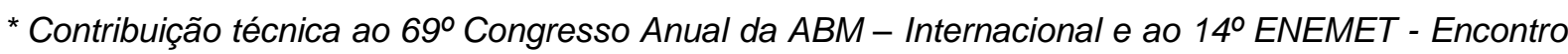
Nacional de Estudantes de Engenharia Metalúrgica, de Materiais e de Minas, 21 a 25 de julho de 2014, São Paulo, SP, Brasil.
} 


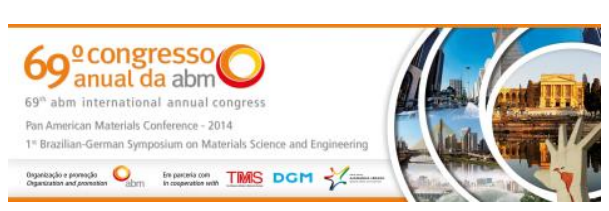

3 Béjar MA, Moreno E. Abrasive wear resistance of boronized carbon and low-alloy steels. Journal of Materials Processing Technology, 2006; 173: 352-358.

$4 \mathrm{Mu} \mathrm{D}$, Shen B, Zhao X. Effects of boronizing on mechanical and dry-sliding wear properties of CoCrMo alloy. Materials and Design, 2010; 31(8): 3933-3936.

5 Campos I, Ramírez G, Villa Velázquez C, Figueroa U, Rodríguez G. Study of microcracks morphology produced by Vickers indentation on AISI 1045 borided steels. Materials Science and Engineering A, 2008; 475: 285-292.

6 Campos-Silva I, Ortiz-Domínguez M, López-Perrusquia N, Meneses-Amador A, Escobar-Galindo R, Martínez-Trinidad J. Characterization of AISI 4140 borided steels. Applied Surface Science, 2010; 256: 2372-2379.

7 Krelling A, Dallmann J, Milan JC, Costa CE. Estudo do comportamento tribológico do aço AISI $\mathrm{H} 13$ submetido a diferentes tratamentos superficiais. In: Anais do $67^{\circ}$ Congresso Internacional da ABM. Anais... . 2012. Rio de Janeiro - RJ.

8 Morozo MA, Krelling AP, Milan JC, Costa CE. Microabrasão do aço AISI H13 com e sem boretação. In: Anais do $68^{\circ}$ Congresso Internacional da ABM. Anais... . 2013. Belo Horizonte - MG.

9 Jain V, Sundararajan G. Influence of the pack thickness of the boronizing mixture on the boriding of steel. Surface and Coatings Technology, 2002; 149: 21-26.

10 SAHIN, S. Effects of boronizing process on the surface roughness and dimensions of AISI 1020 , AISI 1040 and AISI 2714. Journal of Materials Processing Technology, 2009; 209: 1736-1741.

11 Petrova RS, Suwattananont N. Surface modification of ferrous alloys with boron. Journal of Etectronic Materials, 2005; 34(5): 575-582.

12 Aghaie-Khafri M, Mohamadpour Nzar Abady MA study of chromo-boronizing on DIN 1.2714 steel by duplex surface treatment. JOM, 2012; 64(6): 694-701.

13 Pertek $A$, Kulka M. Characterization of complex $(B+C)$ diffusion layers formed on chromium and nickel-based low-carbon steel. Applied Surface Science, 2002; 202: 252260.

14 Forati Rad H, Amadeh A, Moradi H. Wear assessment of plasma nitrided AISI H11 steel. Materials and Design, 2011; 32(5): 2635-2643.

15 Basso RL, Pastore HO, Schmidt V, Baumvol IJ, Abarca SA, Souza FS et al. Microstructure and corrosion behaviour of pulsed plasma-nitrided AISI H13 tool steel. Corrosion Science, 2010; 52(9): 3133-3139.

16 Akhtar SS, Arif AF, Yilbas BS. Evaluation of gas nitriding process with in-process variation of nitriding potential for AISI $\mathrm{H} 13$ tool steel. International Journal of Advanced ManufacturingTechnology, 2010; 47: 687-698.

17 Franco E, Santos JL, Pereira JB, Costa CE, Milan JC. Comportamento tribológico do aço rápido $\mathrm{M} 2$ fabricado por metalurgia do pó e tratado por diferentes métodos de nitretação. In: Anais do $66^{\circ}$ Congresso Internacional da ABM. Anais... . p.1695-1706, 2011. São Paulo - SP.

18 Milan JC, Almeida EA, Oliveira RD, Pereira JB, Costa CE. Efeito de diferentes métodos de nitretação: sólida, gasosa e a plasma sobre o comportamento ao desgaste de aços ferramentas. In: Anais do 64ํㅡㄹ Congresso Anual da ABM. Anais... . 2009. Belo Horizonte, MG.

19 Maragoudakis NE, Stergioudis G, Omar H, Pavlidou E, Tsipas DN. Boro-nitriding of steel US 37-1. Materials Letters, 2002; 57: 949 - 952.

20 American Society for Testing and Materials ASTM. Standard Test Method for Wear Testing with a Pin-on-Disk Apparatus. Reapproved,2010, p. 1-5.

\footnotetext{
* Contribuição técnica ao $69^{\circ}$ Congresso Anual da ABM - Internacional e ao 14ํㅡㄹ ENEMET - Encontro Nacional de Estudantes de Engenharia Metalúrgica, de Materiais e de Minas, 21 a 25 de julho de 2014, São Paulo, SP, Brasil.
} 